\title{
A study of alpha capture cross-sections of ${ }^{112} \mathrm{Sn}$
}

\author{
N. Özkan ${ }^{1, a}$, G. Efe ${ }^{1}$, R.T. Güray ${ }^{1}$, A. Palumbo ${ }^{2}$, M. Wiescher ${ }^{2}$, J. Görres ${ }^{2}$, H.-Y. Lee ${ }^{2}$, Gy. Gyürky ${ }^{3}$, E. Somorjai ${ }^{3}$, \\ and Zs. Fülöp ${ }^{3}$ \\ 1 Kocaeli University, Department of Physics, 41380 Umuttepe, Kocaeli, Turkey \\ 2 University of Notre Dame, Notre Dame, IN, 46556 USA \\ 3 Institute of Nuclear Research (ATOMKI) P.O. Box 51, H-4001 Debrecen, Hungary
}

Received: 1 August 2005 /

Published online: 10 March 2006 - (c) Società Italiana di Fisica / Springer-Verlag 2006

\begin{abstract}
The ${ }^{112} \operatorname{Sn}(\alpha, \gamma){ }^{116}$ Te reaction cross-section has been measured to test the applicability of statistical models, especially NON-SMOKER in the energy range of importance for the astrophysical p-process nucleosynthesis. The measurements were carried out at the Notre Dame FN Tandem Van de Graaff accelerator by means of the activation method. Enriched self-supporting foils were irradiated with alpha beams over the alpha bombarding energy range of $8 \mathrm{MeV}$ to $12 \mathrm{MeV}$ in $0.5 \mathrm{MeV}$ steps. The induced activity was measured with a pair of large volume Ge Clover detectors in close geometry to maximize the detection efficiency. The preliminary results are compared with recent statistical model predictions using the code NON-SMOKER.
\end{abstract}

PACS. 25.40.Lw Radiative capture - 26.30.+k Nucleosynthesis in novae, supernovae, and other explosive environments - 82.20.Pm Rate constants, reaction cross sections, and activation energies

\section{Introduction}

The elements heavier than iron are mainly synthesized by mechanisms referred to as the slow neutron capture (s-process) and the rapid neutron capture (r-process). An additional mechanism, the p-process, is responsible for the production of the observed rare abundances of the protonrich stable nuclides in the mass range $A \geq 74$. These so-called p-nuclei are shielded by stable nuclei from the production via the $\mathrm{r}$ - and s-process.

Early theories exist for the production of the p-nuclei. One of the earliest was proposed by $\mathrm{B}^{2} \mathrm{FH}$ [1] who suggested that the p-process was a series of $(\mathrm{p}, \gamma)$ reactions on $\mathrm{r}$ - and s-process seeds. It was also suggested by Ito that the light p-nuclei could be synthesized at a high-temperature condition $\left(T_{9} \sim 3\right)$ from seeds by $(\mathrm{p}, \gamma)$ reactions while the heavy p-nuclei could be produced at a lower temperature $\left(T_{9} \sim 2.5\right)$ by $(\gamma, \mathrm{n})$ reactions $[2,3]$.

Current theories include synthesis from the destruction of pre-existing s- or r-nuclides by different combinations of $(\mathrm{p}, \gamma)$ captures, $(\gamma, \mathrm{n}),(\gamma, \mathrm{p})$ or $(\gamma, \alpha)$ reactions. Generally, p-nuclei are thought to be synthesized from an unstable progenitor through a chain of $\beta$-decays [4].

In the thermonuclear model, the p-process proceeds via a series of photodisintegration reactions, $(\gamma, \mathrm{n}),(\gamma, \mathrm{p})$ and $(\gamma, \alpha)$ on an existing heavy s- and r-seed in the tempera-

\footnotetext{
a e-mail: nozkan@kou.edu.tr
}

ture range $2-3 \times 10^{9} \mathrm{~K}$. The nuclides are driven by subsequent $(\gamma, \mathrm{n})$ reactions to the neutron-deficient side where $(\gamma, \mathrm{n})$ and $(\mathrm{n}, \gamma)$ reactions are in equilibrium. The reaction path is then dominated by the $(\gamma, \mathrm{p})$ and/or $(\gamma, \alpha)$ reactions $[5,6]$. It is for this reason that radiative alpha capture reactions are of particular importance to the p-process.

Possible astrophysical scenarios for the p-process under consideration are massive stars in their pre-supernova or Type-II supernova phases, as well as their Type-Ib/Ic stages, and white dwarfs exploding as Type-Ia supernova in binary systems. Detailed p-process scenarios have been reviewed by Arnould and Goriely [7].

In comparison to the s- and r-, not much study is devoted to the p-process even though many discrepancies exist. In particular, the theoretical predictions of p-nuclei are frequently inconsistent with their observed abundances [7]. These abundance predictions are based on complex network calculations performed on thousands of nuclear reactions involving stable as well as unstable proton-rich nuclei within a given astrophysical environment. However, only very few of the associated reaction rates in the region of interest have been measured. The relevant reactions that have been investigated in the mass region beyond $A=100$ include $(\alpha, \gamma)$ reactions on targets of ${ }^{106} \mathrm{Cd}[8],{ }^{112} \mathrm{Sn}[9],{ }^{139} \mathrm{La}$ [10], and ${ }^{144} \mathrm{Sm}$ [11]. Due to the lack of experimental data, p-process studies are based mostly on Hauser-Feshbach statistical models to predict the reaction rates. Therefore, it is crucial to determine 
the cross-sections experimentally in order to test the reliability of the input parameters such as optical model potentials and nuclear level densities.

The primary aim of this paper is to extend the experimental database for simulation of the p-process by measuring the $(\alpha, \gamma)$ cross-sections on the p-nucleus ${ }^{112} \mathrm{Sn}$. The photon-induced reaction cross-sections ${ }^{116} \mathrm{Te}(\gamma, \alpha)^{112} \mathrm{Sn}$ $(Q=0.928 \mathrm{MeV})$ can also be calculated by using the detailed balance theorem. In order to test the applicability of statistical models, the deduced cross-section measurements are compared with one of the statistical models, NON-SMOKER [12]. A discussion of experimental procedure and a presentation of preliminary results follows.

\section{Experimental technique}

The cross-section of ${ }^{112} \mathrm{Sn}(\alpha, \gamma){ }^{116} \mathrm{Te}$ has been measured via the activation technique at the FN Tandem Van de Graaff accelerator at the University of Notre Dame, USA in the energy range $8-12 \mathrm{MeV}$. These energies are particularly interesting since they are relevant to the Gamow window for the high-temperature environments.

The activation method involves bombarding a stable isotope with projectiles to produce a radioactive species and the measurement of the residual radioactivity of the produced isotopes after the irradiation is stopped. This method provides a total cross-section for primary $\gamma$-ray transitions to particle bound states (which lead to the formation of the radioactive species in its ground state).

\subsection{Targets}

Isotopically enriched ${ }^{112} \mathrm{Sn}$ targets in the form of thin selfsupporting foils of $2.2 \mathrm{mg} / \mathrm{cm}^{2}$ were used for the measurements. The highly enriched $(99.60 \%){ }^{112}$ Sn targets were prepared at Argonne National Laboratory via mechanical rolling. Metal ingots of enriched Sn are placed in a stainless steel pack in a rolling mill and initially rolled very slowly to prevent cracks. They are subsequently rotated by $90^{\circ}$ each time while slowly tightening the plate gap until a uniform thickness is achieved [13].

\subsection{Irradiation of the target}

${ }^{112} \mathrm{Sn}$ targets were irradiated with an alpha beam in the energy range between 8 and $12 \mathrm{MeV}$ in energy steps of $0.5 \mathrm{MeV}$.

A diagram of the experimental setup for the target irradiation is shown in fig. 1 . In order to get an accurate measurement of the total number of charged particles hitting the target during the irradiation runs, the entire target chamber was designed as a Faraday cup (which was isolated from the rest of the beam line). The beam current was recorded in real time with a current integrator in time steps of $32 \mathrm{~s}$, allowing fluctuations in the beam to be

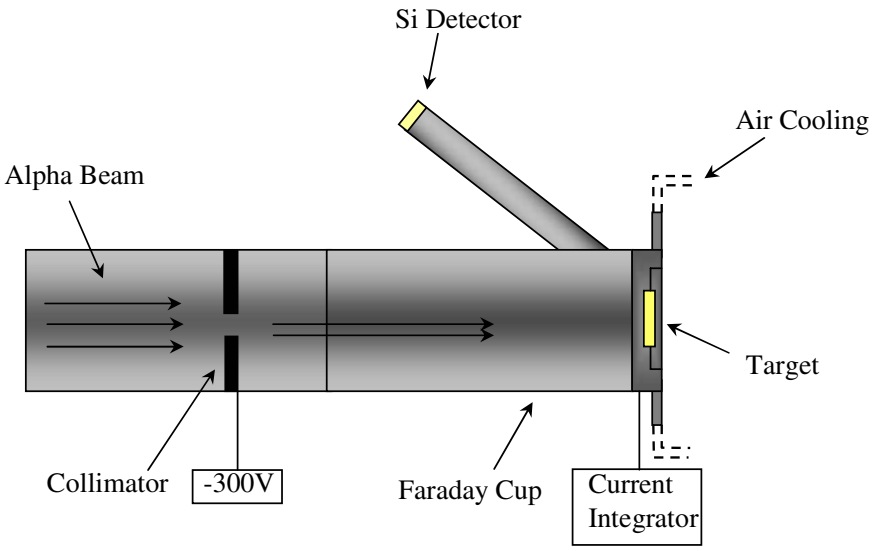

Fig. 1. A drawing of the components used in the beam line during the irradiation. The target was placed at the end of the beam line.

monitored. Throughout the irradiations with different $\alpha$ beam energies, the typical current recorded was between 100 and $250 \mathrm{nA}$.

Electric suppression was employed to suppress the secondary electron emission from the target by a bias voltage of $-300 \mathrm{~V}$. Due to the relatively low melting point of ${ }^{112} \mathrm{Sn}$ and the thick targets, the target holder was air cooled during the irradiation to prevent target degradation. In addition, the target stability was also monitored online by detecting the backscattered alphas from the target using a collimated Si surface barrier detector at 135 degrees. The Si detector was calibrated using a mixed alpha source.

The length of irradiation was chosen based on the halflife of the $(\alpha, \gamma)$ activation product. The typical irradiation time was $\sim 8 \mathrm{~h}(3 \times$ half-life $)$ for low-energy measurements due to the steeply decreasing cross-sections at low beam energies.

\subsection{Measurement of the residual radioactivity}

After each irradiation, the target was taken to a remote low-background counting area. The counting setup was constructed in an area isolated from the target room so the detectors would not be saturated by the inevitable large flux of radiation produced during irradiation. Another advantage of having a separate counting area is that we were able to obtain a larger solid angle by utilizing a close geometry for the target and the detectors.

The detection system was composed of two Clover Ge detectors (Clover 1 and Clover 2), each detector having four HPGe crystals. Figure 2 shows the arrangement of detectors and the irradiated sample in the measurement. The two Clovers were utilized as a single detection unit, which is said to be operated in "direct" mode as described by ref. [14]. This implies that the photopeak detection efficiency of the system was the sum of each photopeak efficiency of one crystal ( 8 crystals in all). The nearly $4 \pi$ detection geometry offers relatively high efficiency enabling the detection of low-energy gamma peaks at low 


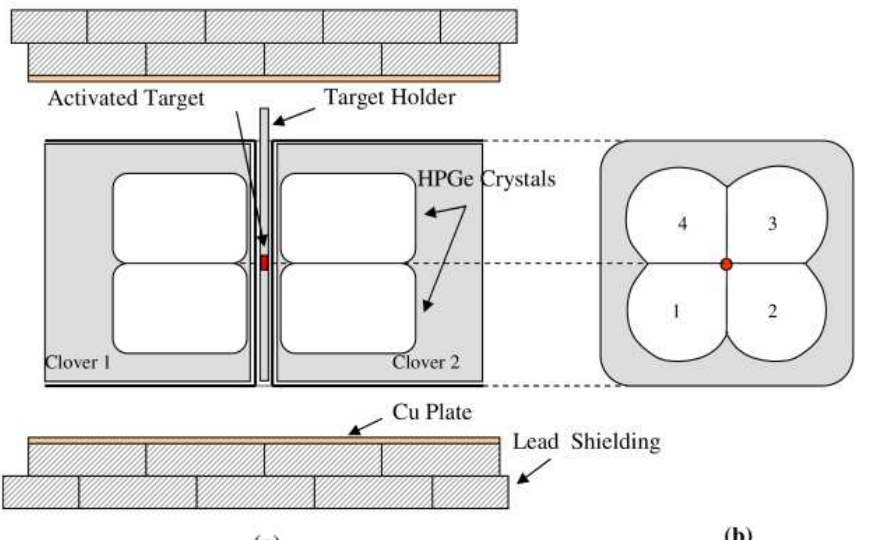

(a)

(b)

Fig. 2. The scheme of experimental setup used to measure the induced $\gamma$-ray activity. (a) Low-background counting area ensured by $4 \pi$ lead bricks and $\mathrm{Cu}$ plates. (b) Target position viewed from the front window of the Clover 2.

irradiation energies. Due to this geometry, corrections for angular-distribution effects were negligible.

In order to measure the induced $\gamma$-ray activity, the Clover Ge detectors were placed face to face in close geometry. The distance between the ends of the detectors was fixed at $4.9 \mathrm{~mm}$ for reproducibility of the counting geometry. To reduce the room background, the detectors were shielded with $5 \mathrm{~cm}$ of $\mathrm{Pb}$ and an inner $\mathrm{Cu}$ lining of $3 \mathrm{~mm}$. After each irradiation, the activated target was placed at the center of the detectors and positioned at their common axis as shown in fig. 2. Depending on the count rate of the targets, the decays were observed in a time interval from $20 \mathrm{~m}$ to $8 \mathrm{~h}\left(t_{1 / 2}=2.49 \mathrm{~h}\right)$. For the dead-time correction, the output signal of a fixed-frequency $(100 \mathrm{~Hz})$ pulse generator was also fed into the electronics.

\section{Experimental results}

We were able to measure cross-sections from daughter and granddaughter decays since both the daughter nucleus ${ }^{116} \mathrm{Te}\left(t_{1 / 2}=2.49 \mathrm{~h}\right)$ and the granddaughter nucleus ${ }^{116} \mathrm{Sb}\left(t_{1 / 2}=15.8 \mathrm{~m}\right)$ are radioactive as seen in fig. 3 . The $\gamma$-ray spectrum for the ${ }^{112} \mathrm{Sn}(\alpha, \gamma){ }^{116}$ Te reaction obtained at $12 \mathrm{MeV} \alpha$-beam irradiation is presented in figs. 4 and 5 , as an example of the $\gamma$-ray activities accumulated during and after termination of the activation measurements. The characteristic $\gamma$-transitions of $629 \mathrm{keV}$ and $638 \mathrm{keV}$ in ${ }^{116} \mathrm{Sb}$ populated through the $\beta^{+}$-decay of the reaction product ${ }^{116} \mathrm{Te}$ were used to measure the cross-section in the alpha-beam energy range $9.5-12.0 \mathrm{MeV}$, (fig. 4). For cross-section measurements at lower alpha-beam energies, we used two additional $\gamma$-decay transitions, $932 \mathrm{keV}$ and $1294 \mathrm{keV}$, in ${ }^{116} \mathrm{Sn}$ populated by the ${ }^{116} \mathrm{Sb}$ secondary $\beta^{+}$. decay (fig. 5).

The total error of the measured cross-section values includes two components: statistical error based on counting

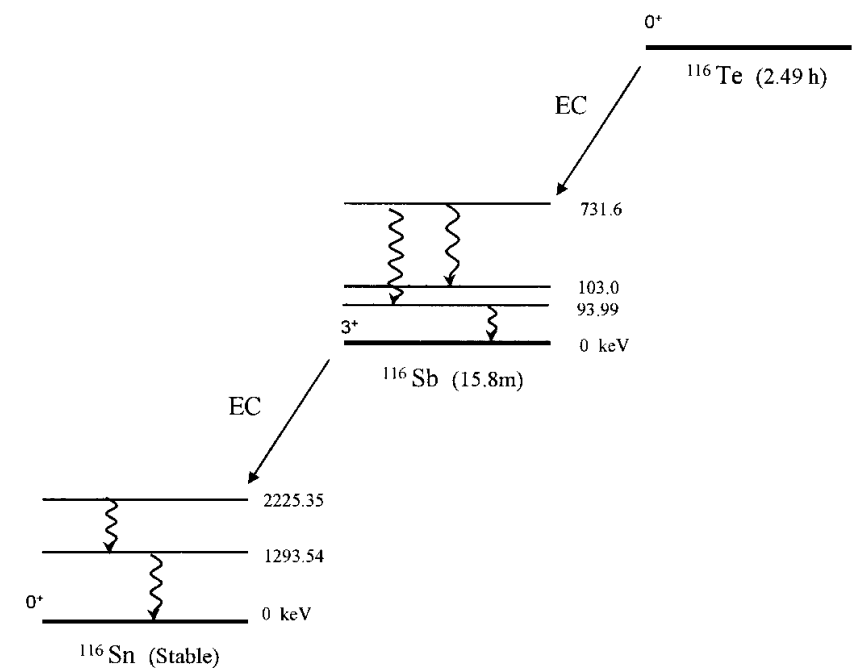

Fig. 3. A simple decay scheme of the residual nucleus, ${ }^{116} \mathrm{Te}$; energies given in $\mathrm{keV}[15]$.

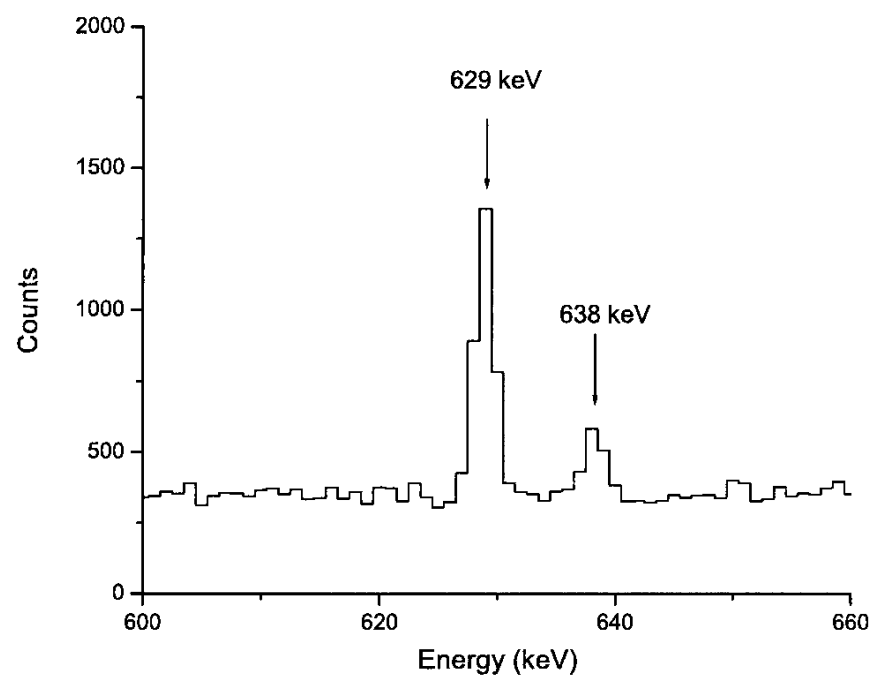

Fig. 4. The $\gamma$-ray spectrum in the relevant energy region obtained after activation with $\alpha$ beams of $12 \mathrm{MeV}$. The $\gamma$ lines from the $\beta^{+}$-decay of the daughter nucleus ${ }^{116} \mathrm{Te}\left(t_{1 / 2}=\right.$ $2.49 \mathrm{~h}$ ) used for cross-section measurements are indicated by arrows.

statistics $(\leq 20 \%)$ and systematical error based on the errors in the procedural techniques such as the error in the efficiency measurement $(10 \%)$, the error in the beam current integration (2\%), and target thickness $(5 \%)$. These components were added in quadrature.

Good agreement was obtained for the cross-sections resulting from the analysis of these four $\gamma$-decay transitions. These cross-sections are shown as a function of center-of-mass energy in fig. 6 in comparison with the predictions of NON-SMOKER calculations [12] (solid line). While good agreement can be observed at higher energies, the experimental data deviate considerably in the lowerenergy range from the theoretical predictions. 


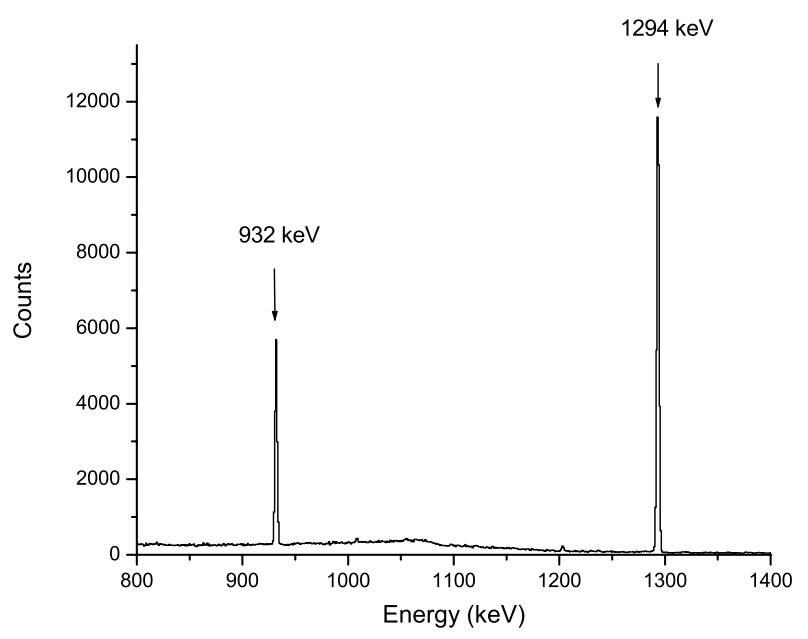

Fig. 5. The $\gamma$-ray spectrum in the relevant energy region obtained after activation with $\alpha$ beams of $12 \mathrm{MeV}$. The $\gamma$ lines from the $\beta^{+}$-decay of the granddaughter nucleus ${ }^{116} \mathrm{Sb}$ $\left(t_{1 / 2}=15.8 \mathrm{~m}\right)$ used for cross-section measurements are also indicated by arrows.

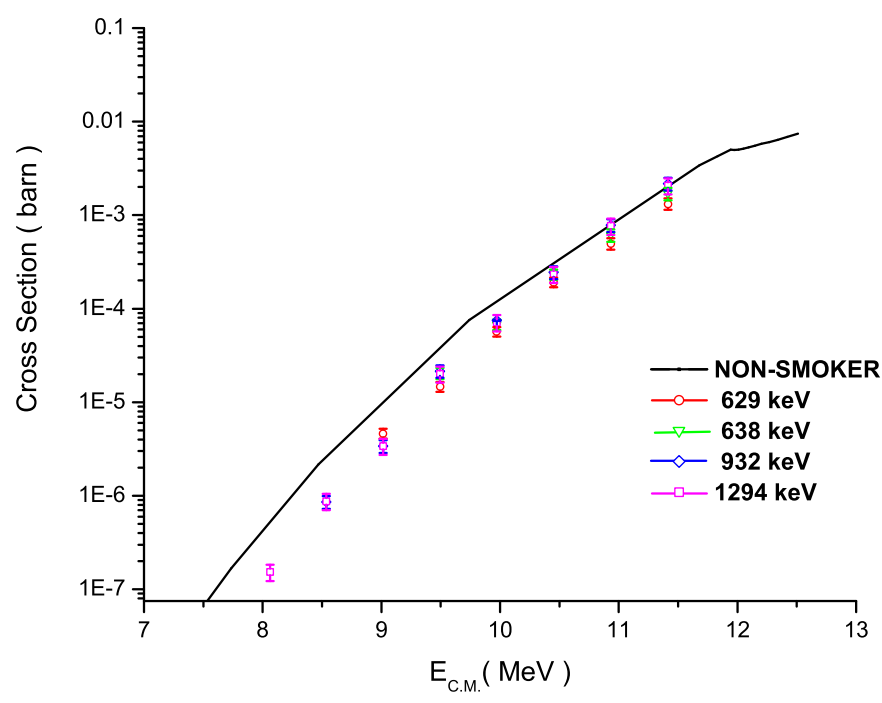

Fig. 6. The cross-sections of $\alpha$-induced reactions on ${ }^{112} \mathrm{Sn}$ as a function of center-of-mass energy. The experimental results (individual points) as well as the predictions of NON-SMOKER calculations are shown [12].

In this work, the cross-sections for ${ }^{112} \mathrm{Sn}(\alpha, \gamma){ }^{116} \mathrm{Te}$ have been measured in an energy range directly relevant to nuclear astrophysics. This energy range spans the Gamow window predicted for this reaction in the hightemperature environments; the Gamow window ranges between $6.8 \mathrm{MeV}$ and $10.2 \mathrm{MeV}$ for $T=3 \times 10^{9} \mathrm{~K}$. Further analysis of these data is in progress, but the present preliminary results are promising. These results will be compared with the statistical model calculations using different input parameters.

It is a pleasure to thank John P. Greene from Argonne National Laboratory for preparing the ${ }^{112} \mathrm{Sn}$ targets for us. This work was supported by The Scientific and Technical Research Council of Turkey TUBITAK - Grant TBAG-U/111 (104T2467) [16], the National Science Foundation NSF - Grant 0434844 and the Joint Institute for Nuclear Astrophysics JINA, PHY02-16783 [17], and The Hungarian Scientific Research Fund Programs OTKA - Grant T 42733, T 49245, F 43408, D 48283. Zs.F. is a Bolyai fellow.

\section{References}

1. E.M. Burbidge, G.R. Burbidge, W.A. Fowler, F. Hoyle, Rev. Mod. Phys. 29, 547 (1957).

2. K. Ito, Prog. Theor. Phys. 26, 990 (1961).

3. D.L. Lambert, Astron. Astrophys. Rev. 3, 201 (1992).

4. R.N. Boyd, Heavy Elements and Related New Phenomena, edited by W. Greiner, R.K. Gupta (World Scientific, 1999) p. 893.

5. S.E. Woosley, W.M. Howard, Astrophys. J. Suppl. 36, 285 (1978).

6. M. Rayet, Astron. Astrophys. 227, 271 (1990).

7. M. Arnould, S. Goriely, Phys. Rep. 384, 1 (2003).

8. Gy. Gyürky et al., Nucl. Phys. A 758, 517c (2005) (preliminary results).

9. N. Özkan, A.St.J. Murphy, R.N. Boyd, A.L. Cole, M. Famiano, R.T. Güray, M. Howard, L. Sahin, J.J. Zack, R. deHaan, J. Görres, M.C. Wiescher, M.S. Islam, T. Rauscher, Nucl. Phys. A 710, 469 (2002).

10. E.V. Verdieck, J.M. Miller, Phys. Rev. 152, 1253 (1967).

11. E. Somorjai et al., Astron. Astrophys. 333, 1112 (1998).

12. T. Rauscher, F.K. Thielemann, At. Data Nucl. Data Tables 79, 427 (2001).

13. J.P. Greene, private communication.

14. S. Dababneh, N. Patronis, P.A. Assimakopoulos, J. Görres, M. Heil, F. Käppeler, D. Karamanis, S. O'Brien, R. Reifarth, Nucl. Instrum. Methods A 517, 230 (2004).

15. R. Firestone, in Table of Isotopes, edited by V. Shirley (Wiley, New York, 1996).

16. http://www . tubitak.gov.tr.

17. http://www. JINAweb. org. 